Received: 1 September 2017

Accepted: 26 January 2018

Published online: 07 February 2018
NTIFIC REP

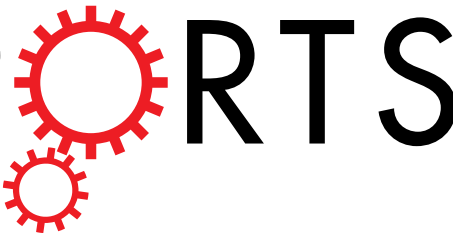

\section{OPEN IRE1 $\alpha$ siRNA relieves endoplasmic reticulum stress-induced apoptosis and alleviates diabetic peripheral neuropathy in vivo and in vitro}

\author{
Weijie Yao, Xinwei Yang, Jiayue Zhu, Biane Gao, Haotian Shi \& Liping Xu
}

Diabetic peripheral neuropathy (DPN) is mainly characterized by demyelination resulted from the apoptosis of the Schwann cell (SCs). Although the exact mechanisms underlying DPN remain unclear, endoplasmic reticulum (ER) stress is strongly implicated in the apoptosis. Under ER stress, activated inositol-requiring kinase $1 \alpha$ (IRE1 $\alpha$ ) unregulated CHOP, phosphorylated JNK and Caspase-12 to aggravate apoptosis-mediated damage of DPN. Therefore, we tested the hypothesis that inhibition of IRE1 $\alpha$ could reduce the ER stress-related apoptosis to relieve DPN. Here, we show that IRE1 $\alpha$ siRNA improved the neurological morphology and function of DPN rats and rescued ER stress-related apoptosis in the sciatic nerve. Additionally, RSC96 cells transfected with IRE1 $\alpha$ siRNA were used as in vitro model of DPN. It was found that IRE1 $\alpha$ siRNA also decreased high glucose-induced apoptosis and inhibited ER stress-related apoptosis in the cells. Altogether, our results suggest that IRE1 $\alpha$ should be considered a potential therapeutic agent for DPN.

Diabetic peripheral neuropathy (DPN), one of the diabetes complications, is a neurodegenerative disease with no treatment options available ${ }^{1}$. Currently, Schwannopathy is considered to be one of the integral factors in the pathogenesis of $\mathrm{DPN}^{2}$. Chronic hyperglycemia induces endoplasmic reticulum (ER) stress, which is the key factor leading to the apoptosis of the Schwann cell (SCs), and contributing to DPN ${ }^{3}$.

ER is the major organelle for the translocation, folding, and post-translational modifications of proteins. Perturbations in ER function, a process named ER stress, trigger unfolded protein response (UPR), a complementary adaptive machinery, organized by three ER transmembrane receptor proteins. Inositol-requiring kinase $1 \alpha($ IRE $1 \alpha)$, double-stranded RNA-activated protein kinase (PKR)-like endoplasmic reticulum kinase (PERK) and activating transcription factor 6 (ATF6) are against ER stress to restore protein homeostasis ${ }^{4}$. If the stress is too severe and excesses the capacity of UPR defense mechanisms, cells switch to apoptotic cell mechanisms ${ }^{5}$.

IRE $1 \alpha$ determines cell fate based on ER stress severity. Under unrelieved ER stress, IRE1 $\alpha$ degrades many ER-localized mRNAs to induce apoptosis. Hyperglycemia and hyperlipidemia in diabetes patients hyperactivate IRE1 $\alpha$ and potentiate ER stress-induced apoptosis ${ }^{6}$. Our previous study has shown that ER stress-induced reactive oxygen species (ROS) is a unified pathological process of DPN in vivo and in vitro, and ROS feedback induces the IRE $1 \alpha$, thereby aggravating $\mathrm{DPN}^{7-9}$. The high sensitivity of IRE1 $\alpha$ to hyperglycemia implies that it plays important role in DPN-induced apoptosis ${ }^{10,11}$. However, how the IRE1 $\alpha$ pathway is mediated in DPN is not fully clarified. In addition, the inhibitor of IRE1 $\alpha$ or IRE1 $\alpha$ gene knockdown has been used to intervene diabetes or other metabolic disorders ${ }^{6,12}$. Therefore, this research will focus on the IRE1 $\alpha$ pathway in DPN rats and the Schwann cells to investigate whether IRE1 $\alpha$ siRNA could relieve DPN as well as the molecular mechanisms underlying ER stress-related apoptosis. IRE1 $\alpha$ is expected to be a potentially valuable target for improving DPN.

In this work we evaluated IRE1 $\alpha$ inhibition to decrease the ER stress-induced apoptosis, using experimental models of DPN. Here, we demonstrate that inhibition of IRE1 $\alpha$ reduces the CHOP, Caspase-12 and phosphorylated JNK related-apoptosis and decreases the demyelination of DPN, potentially leading to new therapeutic approaches.

School of Traditional Chinese Medicine, Capital Medical University, Beijing, China. Weijie Yao and Xinwei Yang contributed equally to this work. Correspondence and requests for materials should be addressed to L.X. (email: xulp@ccmu.edu.cn) 
A

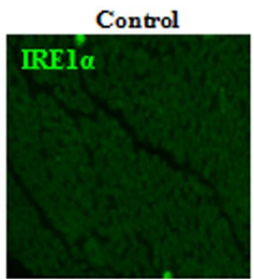

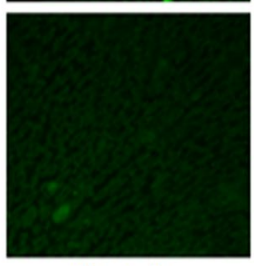

Control+ IREla siRNA
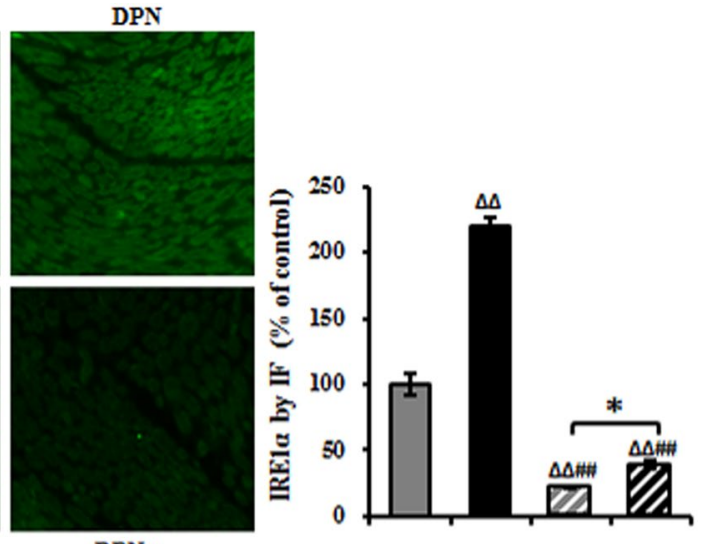

DPN+

IREL $Q$ siRNA

B
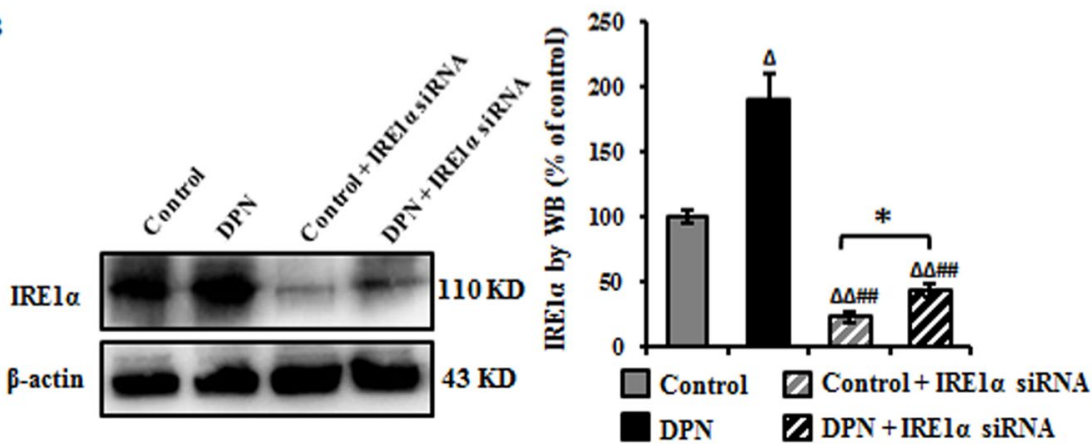

Figure 1. Down-regulation of IRE $1 \alpha$ in the sciatic nerve after intrathecal injection of siRNA. (A) IRE1 $\alpha$ was effectively reduced as measured by immunofluorescence. IRE1 $\alpha$ was detected with an antibody against IRE $1 \alpha$, followed by a fluorescent-labeled secondary antibody (green). Data are expressed as mean \pm SEM as the percentage of the respective controls and analyzed using one-way ANOVA with LSD analysis or unpaired Student's t-test. (B) Representative Western blots using tissue extracts from the sciatic nerve and probed with antibodies against IRE1 $\alpha$. $\beta$-actin was used as loading control. Data are expressed as mean \pm SEM indicated as the percentage of the respective controls and analyzed using Tamhane's T2 analysis or unpaired Student's t-test. ${ }^{\Delta} P<0.05,{ }^{\Delta \Delta} P<0.01$, compared to control; ${ }^{\# \#} P<0.01$, compared to DPN; ${ }^{*} P<0.05$ ( $\mathrm{N}=6$ per group).

\section{Results}

Intrathecal injection siRNA decreased IRE1 $\alpha$ in the sciatic nerve of DPN rats. Our previous study has demonstrated that IRE1 $\alpha$ is overexpressed in the sciatic nerve of high-carbohydrate/high-fat diet and lowdose STZ-induced DPN rats and this result was further verified in this study (Fig. 1A), where overexpressed IRE1 $\alpha$ induced the prolonged ER stress, thus leading to demyelination and nerve dysfunction ${ }^{13}$. Intrathecally injected IRE1 $\alpha$ siRNA was used to further investigate the IRE1 $\alpha$ pathway in DPN. Because this method has not been fully reported in DPN rats, immunofluorescence (IF) staining and Western blotting (WB) were used to examine IRE1 $\alpha$ expression in the sciatic nerve. 3 days after injection, IRE1 $\alpha$ was reduced to $22 \%$ (IF staining) and $24 \%$ (WB), respectively, as compared to IRE1 $\alpha$ siRNA control-transfected group and to $39 \%$ (IF staining) and $44 \%$ (WB) in DPN IRE1 $\alpha$ siRNA transfected group compared to DPN group (Fig. 1). These results suggest that intrathecal injection of IRE1 $\alpha$ siRNA downregulates the expression of IRE1 $\alpha$ in the sciatic nerve.

IRE1 $\alpha$ siRNA improved neurological morphology and function of DPN rats. To verify if IRE $1 \alpha$ siRNA could alleviate DPN, we investigated the morphological and function of the peripheral nerves with a multimodal approach. At the pathological level, as shown in Fig. 2A, the myelin sheath and nerve fibers were reduced to $63 \%$ and $44 \%$, respectively, in DPN rats. In the DPN rats transfected with IRE1 $\alpha$ siRNA the myelin sheath and nerve fibers increased to $82 \%$ and $60 \%$, respectively (Fig. 2B,C). In the DPN rats, there was a significant decrease of the number of cutaneous unmyelinated fibers, as evaluated by intraepidermal nerve fiber density (IENFD) assessment (reduced to 47\%), but after IRE1 $\alpha$ siRNA transfection, the IENFD increased 0.75-fold (Fig. 2D,E). The MNCV and SNCV in DPN rats were reduced compared to control rats, while IRE1 $\alpha$ siRNA treatment increased the MNCV and SNCV compared to DPN rats (Fig. 2F).

IRE1 $\alpha$ siRNA alleviated ER stress-related apoptosis in the sciatic nerve of DPN rats. ER stress activates the UPR signaling pathways that determine cell fate. Remediable ER stress activates adaptive-UPR outputs that favor cell survival, but chronic ER stress activates terminal-UPR (T-UPR) outputs to trigger apoptosis. T-UPR is sufficient to drive IRE1a autophosphorylation, XBP1 mRNA splicing, induction of apoptosis ${ }^{6}$. 
A

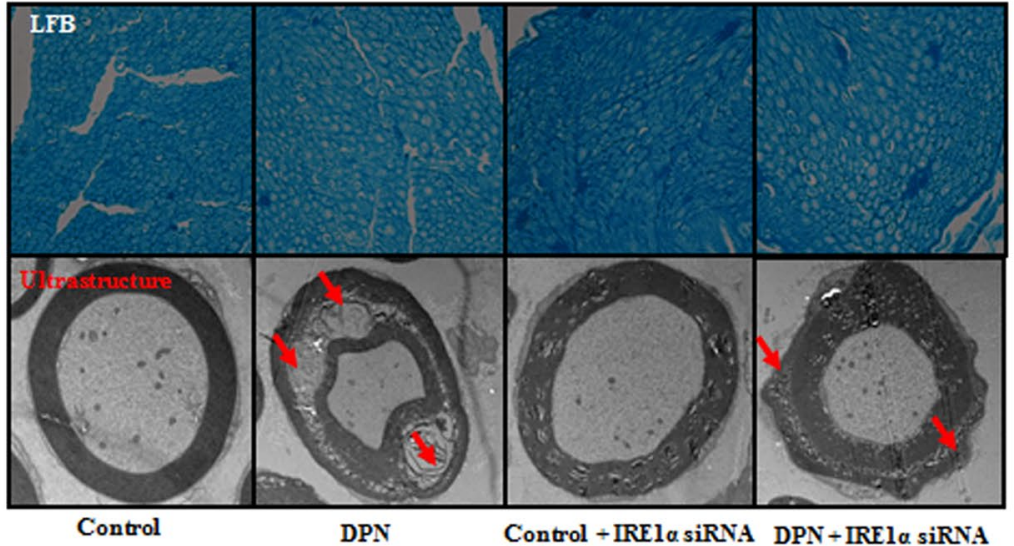

B

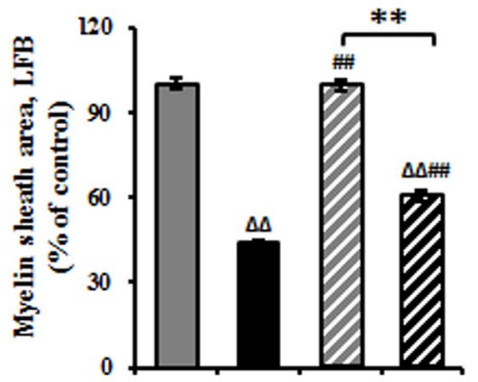

D

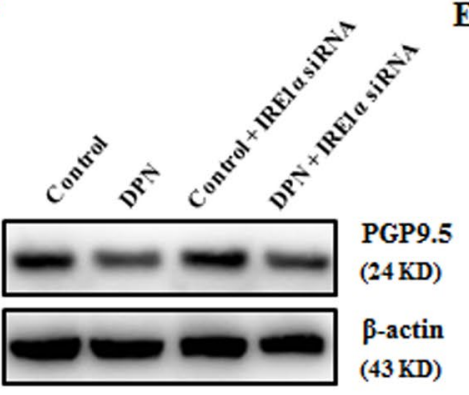

$\mathbf{E}$
C
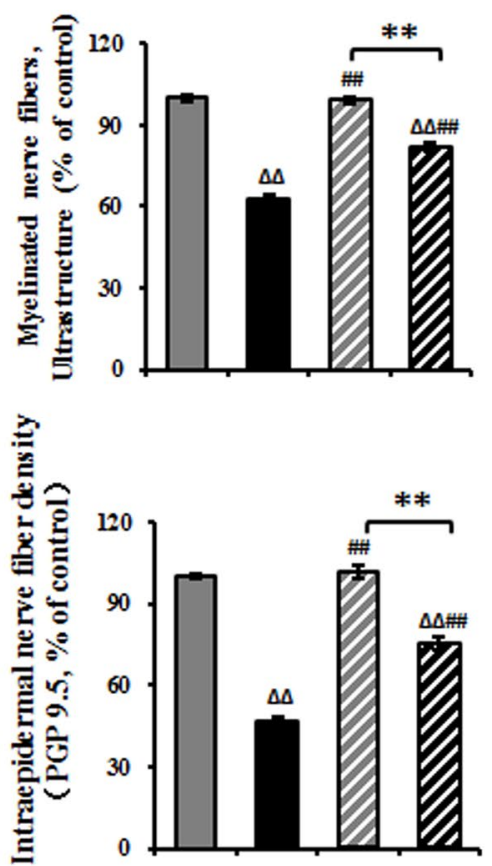

F

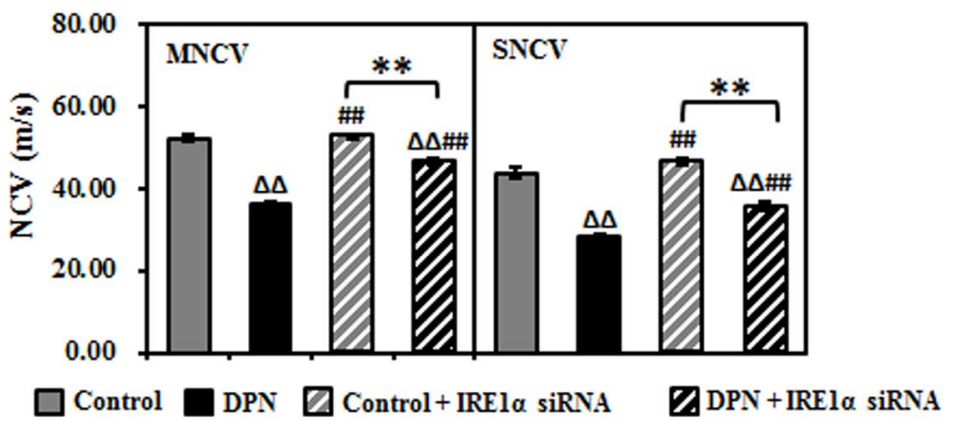

Figure 2. Improved neurological morphology and function of sciatic nerve in DPN rats after the intrathecal injection of IRE1 $\alpha$ siRNA. (A) Transfection of IRE1 $\alpha$ siRNA increased the myelin sheath area and myelinated nerve fibers in DPN rats. Representative pictures of Luxol fast blue (LFB) staining (magnification $40 \times$ ) of myelin sheath and ultrastructure (magnification $6000 \times$ ) of myelinated nerve fibers. (B) Myelin sheath area expressed as mean \pm SEM of the percentage of the respective controls and analyzed using one-way ANOVA with LSD analysis or unpaired Student's t-test. (C) The number of myelinated nerve fibers expressed as mean \pm SEM of the percentage of the respective controls and analyzed using one-way ANOVA with LSD analysis or unpaired Student's t-test. (D) Representative Western blots using tissue extracts from the sciatic nerve and probed with antibodies against PGP9.5. $\beta$-actin was probed as loading control. (E) The intensity of PGP9.5 expressed as mean \pm SEM of the percentage of the respective controls and analyzed using one-way ANOVA with LSD analysis or unpaired Student's t-test. (F) $\mathrm{NCV}$ reported as mean \pm SEM and analyzed using one-way ANOVA with LSD analysis or unpaired Student's t-test. ${ }^{\Delta \Delta} P<0.01$, compared to control; ${ }^{\#} P<0.01$, compared to $\mathrm{DPN} ; * * P<0.01$ (N=6 per group). 
ER stress-induced apoptosis has been reported to be a critical event in DPN, and IRE1 $\alpha$ autophosphorylation resulted from hyperactivation is necessary and sufficient to trigger the apoptosis ${ }^{5}$. We therefore examined the phosphorylation state of IRE1 $\alpha$. IRE1 $\alpha$ became $134 \%$ activation-loop autophosphorylated in DPN rats compared to control rats, where the expression of XBP-1s increased to $117 \%$. While the depletion IRE1 $\alpha$ in the DPN rats down-regulated the expression of P-IRE1 $\alpha$ and XBP- 1 expression by $51 \%$ and $65 \%$, respectively (Fig. 3A). We hypothesized that deactivation of IRE1 $\alpha / \mathrm{XBP}-1$ may inhibit apoptosis in DPN. The TUNEL-positive area in the sciatic nerve of DPN rats increased $40 \%$ compared to control rats. The expression of Cleaved-Caspase- 3 was up-regulated to $150 \%$, while the knock down of IRE1 $\alpha$ in the DPN rats reduced the TUNEL-positive area and expression of cleaved-Caspase- 3 to $108 \%$ and $31 \%$, respectively (Fig. 3B,C).

Apoptosis-related proteins in the IRE1 $\alpha / \mathrm{XBP}-1$ pathway was examined including CHOP, $\mathrm{p}$-JNK and Caspase- $12^{13}$. Results showed that the expression of CHOP, p-JNK and Caspase-12 were increased to $127 \%$, $170 \%$, and $238 \%$ in the DPN rats compared to control rats, and the depletion of IRE1 $\alpha$ in the DPN rats reduced the levels of CHOP, p-JNK and Caspase-12 to 47\%, 75\%, and 63\%. However, IRE1 $\alpha$ siRNA didn't affect the GRP78 expression. CHOP has been shown to down-regulate Bcl-2 while increase the translocation of Bax from cytosol to mitochondria, which consequently triggers apoptosis. In the DPN rats, the expression of Bcl-2 and Bax increased to $122 \%$ and $143 \%$, respectively, compared to control rats. The depletion of IRE1 $\alpha$ in DPN rats reduced the expression of Bax to $64 \%$, while increased Bcl-2 to $186 \%$, as compared to control (Fig. 3B,C).

IRE1 $\alpha$ siRNA decreased high glucose-induced hyperactivation of IRE1 $\alpha / X B P-1 s$ in RSC96 cells. Our previous study has demonstrated that high glucose in the Schwann cell-rich sciatic nerve plays a key role in the pathogenesis of DPN ${ }^{7}$. To further confirm the effects of IRE1 $\alpha$ siRNA on DPN, high glucose-treated Schwann cells (SCs) were used for investigation. For this purpose, RSC96 cells were transfected with IRE1 $\alpha$ siRNA followed by exposure to high level of glucose $(150 \mathrm{mM})$ for 24 and 48 hours. IRE1 $\alpha$ was persistently up-regulated to $138 \%$ and $194 \%$ after the cells were incubated with $150 \mathrm{mM}$ glucose for 24 and 48 hours. Transfection of IRE1 $\alpha$ siRNA down-regulated the expression to $32 \%$ and $48 \%$ in $25 \mathrm{mM}$ glucose and $150 \mathrm{mM}$ glucose at $24 \mathrm{~h}$; and to $40 \%$ and $69 \%$ at $48 \mathrm{~h}$, respectively, suggesting that the expression of IRE1 $\alpha$ in RSC 96 cells was downregulated (Fig. 4A).

The expression of p-IRE $1 \alpha$ and XBP-1s treated with $150 \mathrm{mM}$ glucose was sustainably increased to $165 \%, 159 \%$ (p-IRE $1 \alpha$ ) and 124\%, 149\% (XBP-1s), respectively, compared to untreated cells at 24 and $48 \mathrm{~h}$, while the transfection of IRE $1 \alpha$ siRNA reduced the expression of p-IRE1 $\alpha$ and XBP-1s to $48 \%, 70 \%$ (p-IRE1 $\alpha$ ) and $71 \%, 49 \%$ (XBP-1s) respectively. On the contrast, the expression of GRP78 was not affected (Fig. 4B,C).

IRE1 $\alpha$ siRNA decreased high glucose-induced apoptosis and $\mathrm{Ca}^{2+}$ level in RSC96 cells. Flow cytometry showed that cells treated with $150 \mathrm{mM}$ glucose had 1.73 -fold increase in apoptosis as compared with those treated with $25 \mathrm{mM}$ glucose after 24 hours of exposure, while the pretreatment of IRE1 $\alpha$ siRNA reduced the increase to $122 \%$. After 48 hour exposure with $150 \mathrm{mM}$ glucose, the apoptosis increase to $370 \%$ as compared with $25 \mathrm{mM}$ glucose, while the pretreatment of IRE1 $\alpha$ siRNA decreased the increase to $214 \%$ (Fig. 5A,C). We further studied whether IRE1 $\alpha$ knock down could affect the production of intracellular $\mathrm{Ca}^{2+}$ in RSC96 cells. FACS analysis showed that $\mathrm{Ca}^{2+}$ level increased to $206 \%$ and $207 \%$ after incubation in $150 \mathrm{mM}$ glucose for $24 \mathrm{~h}$ and $48 \mathrm{~h}$ as compared with $25 \mathrm{mM}$ glucose. However, the pretreatment of IRE1 $\alpha$ siRNA redued the $\mathrm{Ca}^{2+}$ production to $161 \%$ and $150 \%$ at $24 \mathrm{~h}$ and $48 \mathrm{~h}$ compared with $150 \mathrm{mM}$ glucose (Fig. 5B,D).

IRE1 $\alpha$ siRNA inhibited high glucose-induced ER stress-related apoptosis in RSC96 cells. To characterize the apoptosis after exposure to high glucose, we measured the expression of CHOP, p-JNK and Caspase-12 and cleaved-Caspase-3. Western analyses showed that after exposure to high glucose for 24 hours and 48 hours, the expression of CHOP was up-regulated to $126 \%$ and $159 \%$, respectively, and IRE $1 \alpha$ siRNA transfection reduced the expression to $64 \%$ and $72 \%$, respectively. The expression of p-JNK was up-regulated to $126 \%$ and $162 \%$ and IRE $1 \alpha$ siRNA transfection reduced the expression to $59 \%$ and $51 \%$. The expression of Caspase-12 was up-regulated to $118 \%$ and $141 \%$ and IRE1 $\alpha$ siRNA transfection reduced the expression to $56 \%$ and $76 \%$. The expression of cleaved-Caspase-3 was up-regulated to $143 \%$ and $146 \%$ and IRE1 $\alpha$ siRNA transfection reduced the expression to $69 \%$ and $61 \%$. We found that Bax was up-regulated as compared with untreated cells, whereas IRE1 $\alpha$ siRNA transfection decreased the expression of Bax and further increased the level of Bcl-2 (Fig. 6). Taken together, these observations suggest that IRE1 $\alpha$ siRNA specifically inhibits ER stress-induced apoptosis after exposure to high glucose.

\section{Discussion}

Our data show that intrathecal injection of IRE1 $\alpha$ siRNA resulted in about 77\% and $65 \%$ reduction of apoptosis in rats and the SCs, respectively; inhibition of IRE1 $\alpha$ improved the demyelination of sciatic nerve, enhanced the $\mathrm{MNCV}$ and SNCV, as well as intraepidermal nerve fiber density. In in vitro experiment, IRE1 $\alpha$ siRNA transfection inhibited apoptosis and $\mathrm{Ca}^{2+}$ levels in the SCs. These might be attributed to down-regulation of IRE1-related apoptosis proteins including CHOP and p-JNK in DPN.

Hyperglycemia and dyslipidemia have been identified as activators of ER stress in DPN ${ }^{14}$. Here, high-carbohydrate/high-fat diet and low-dose streptozotocin were used to construct a DPN rat model. Our previous study has demonstrated that in DPN rats the levels of total cholesterol, triglyceride and low-density lipoprotein are increased and the rats become insulin resistant ${ }^{15}$. The characteristic features of DPN include demyelination and axonal atrophy, which lead to NCV deficiency. Schwannopathy is an integral factor in the pathogenesis of $\mathrm{DPN}^{2}$. SC dysfunction has direct effects on neuronal function as a result of myelin disruption, demyelination, and changes in NCV. SCs are the glial cells responsible for producing the myelin sheath in the PNS and are highly susceptible to ER stress, because ER is the major organelle for controlling the production of 

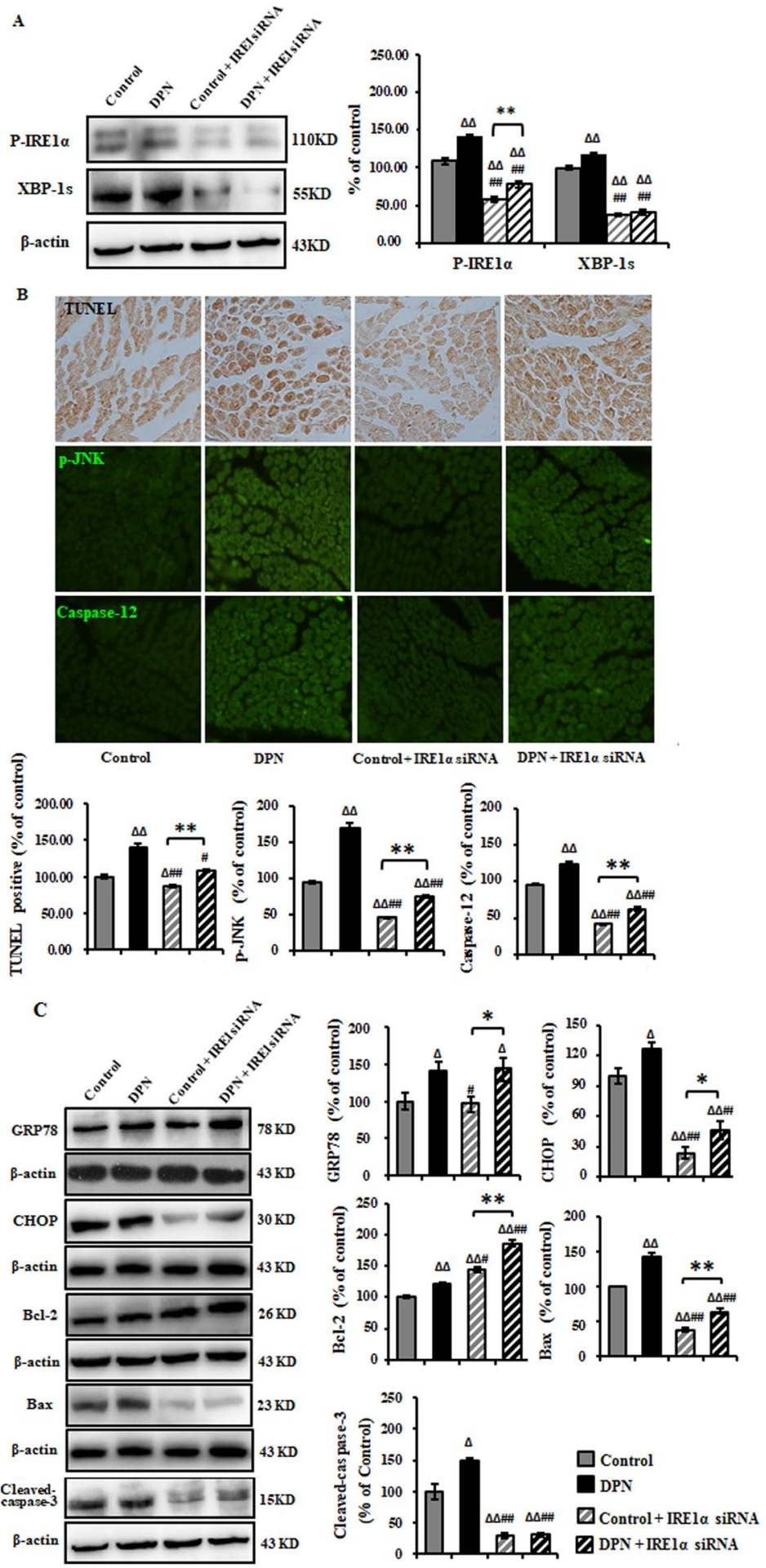

Figure 3. Inhibition of ER stress-related apoptosis in the sciatic nerve of DPN rats by intrathecal injection of IRE1 $\alpha$ siRNA. (A) Representative Western blots using tissue extracts from the sciatic nerve and probed with antibodies against P-IRE1 $\alpha$ and XBP-1s. $\beta$-actin was probed as loading control. The intensity is expressed as mean \pm SEM of the percentage of the respective controls and analyzed using one-way ANOVA with LSD analysis or unpaired Student's t-test. (B) TUNEL, p-JNK, and Caspae-12 were effectively eliminated as measured by immunohistochemistry method. Data are expressed as mean \pm SEM of the percentage of the respective controls and analyzed using one-way ANOVA with Tamhane's T2 analysis or unpaired Student's t-test. (C) Representative Western blots using tissue extracts from the sciatic nerve and probed with antibodies against GRP78, CHOP, Bcl2 , Bax and Cleaved-Caspase-3. $\beta$-actin was probed as loading control. Results of are expressed as mean \pm SEM indicated as percentage of the respective controls and analyzed using one-way ANOVA with LSD analysis (GRP78, Cleaved-Caspase-3, CHOP) or Tamhane's T2 analysis (Bcl-2, Bax) or unpaired Student's t-test. ${ }^{\Delta} P<0.05$, ${ }^{\Delta} \mathrm{P}<0.01$, compared to control; ${ }^{\#} P<0.05,{ }^{\# \#} P<0.01$, compared to $\mathrm{DPN} ;{ }^{*} P<0.05$, ${ }^{* *} P<0.01$ (N=6 per group). 
A
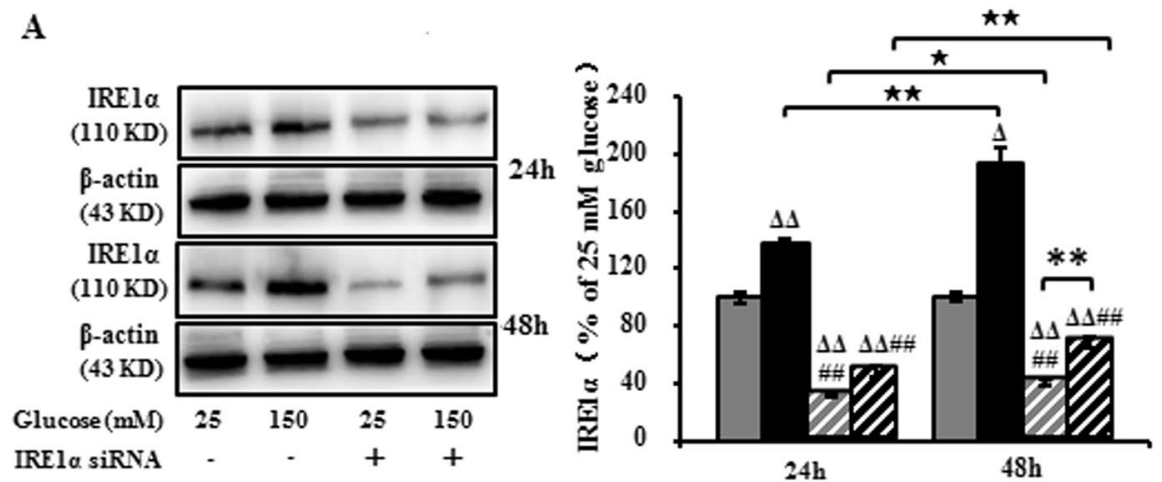

B

24h

$48 \mathrm{~h}$
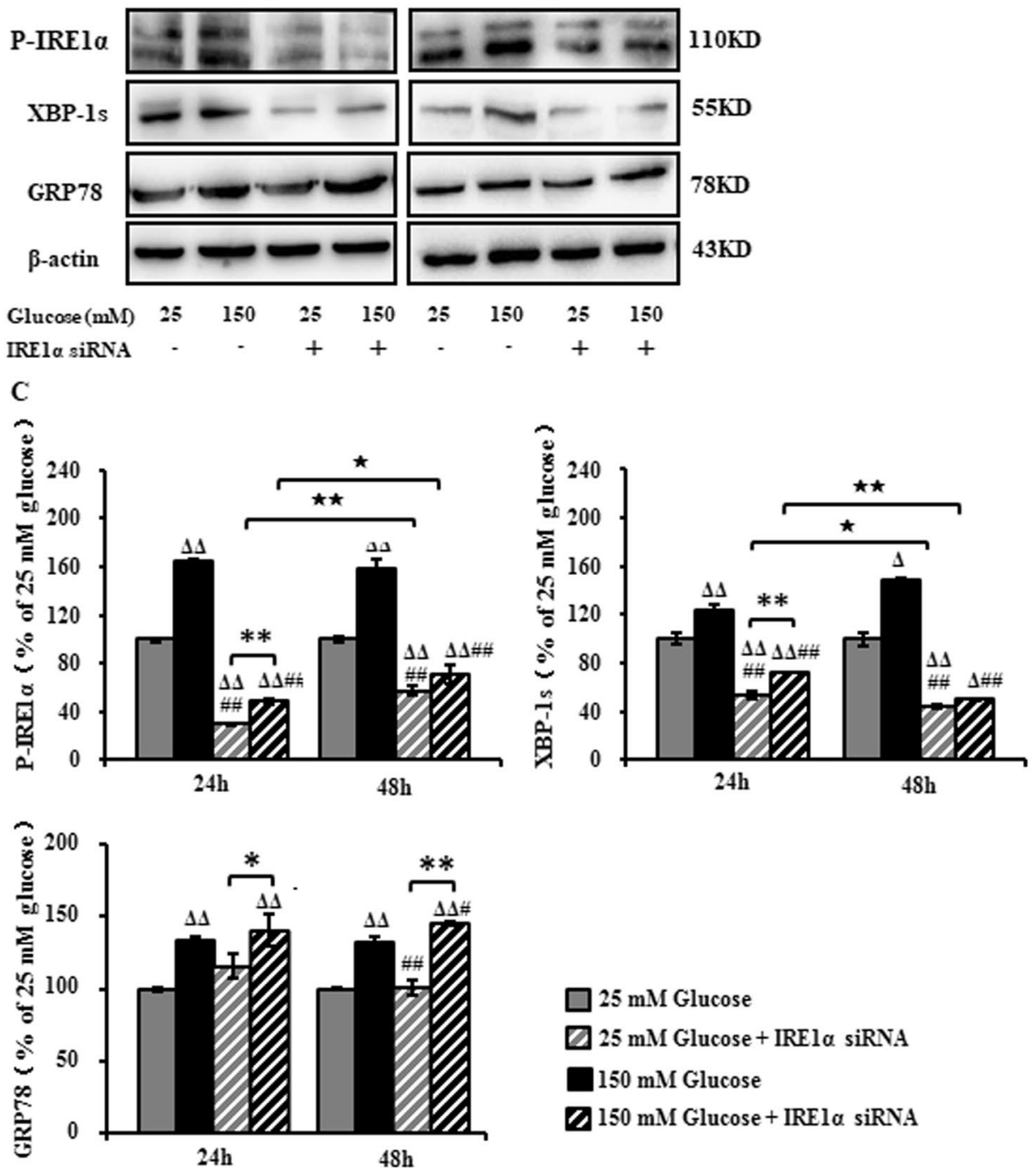

Figure 4. Expression of IRE1 $\alpha$, XBP-1s and GRP78 in RSC96 cells transfected with IRE1 $\alpha$ siRNA and exposed to high glucose. (A-C) Representative Western blots using RSC96 cells and probed with antibodies against IRE1 $\alpha$, P- IRE1 $\alpha$, XBP-1s and GRP78. Results are expressed as mean \pm SEM of the percentage of the respective $25 \mathrm{mM}$ glucose group and analyzed using one-way ANOVA analysis. ${ }^{\Delta} P<0.05, \Delta \Delta P<0.01$, compared to $25 \mathrm{mM}$ glucose; ${ }^{\sharp} P<0.05,{ }^{* \#} P<0.01$, compared to $150 \mathrm{mM}$ glucose; ${ }^{\star} P<0.05,{ }^{\star \star} P<0.01$, Student's unpaired t-test compared to 24 hours' time point. $* P<0.05, * * P<0.01$ ( $\mathrm{n}=4$ per group).

cholesterol and lipid-membrane biosynthesis ${ }^{16,17}$. Therefore, high glucose-treated SCs were used for investigation. Our in vivo results reported here and in our previous publication have demonstrated that SC apoptosis results in demyelination, and then reduces the NCV and IENFD. 

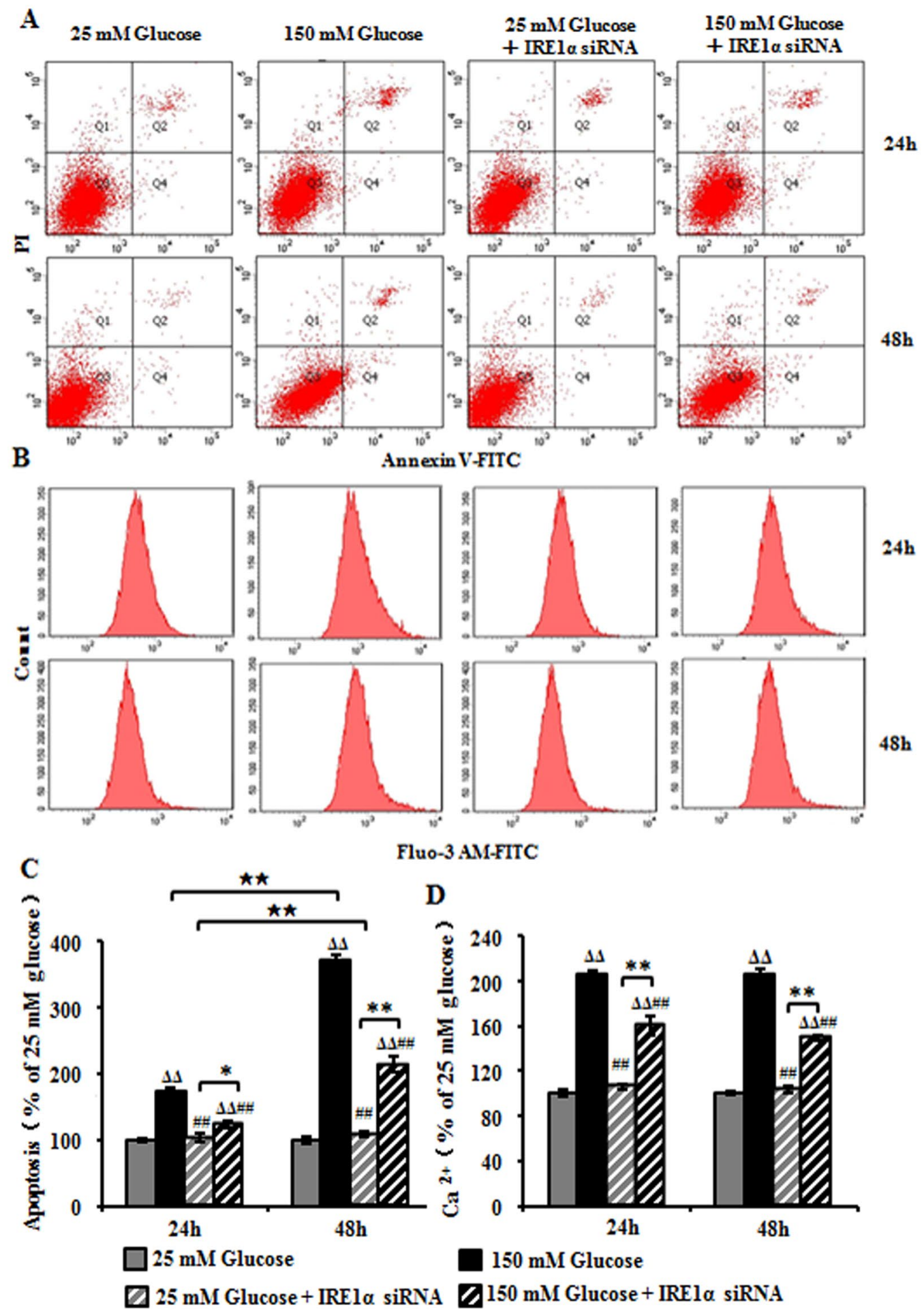

Figure 5. High glucose-induced apoptosis and $\mathrm{Ca}^{2+}$ levels in RSC96 cells after IRE1 $\alpha$ siRNA transfection. (A) FACS apoptosis assay results using FITC-Annexin V and PI staining at $24 \mathrm{~h}$ and $48 \mathrm{~h}$. (B) Intracellular $\mathrm{Ca}^{2+}$ production measured with FACS analysis using Fluo-3 AM dye at $24 \mathrm{~h}$ and $48 \mathrm{~h}$. (C) Apoptosis levels indicated as the percentage of the respective $25 \mathrm{mM}$ glucose-treated cells. Data are expressed as mean $\pm \mathrm{SEM}$ and analyzed using one-way ANOVA with LSD analysis. (D) Intracellular $\mathrm{Ca}^{2+}$ levels indicated as the percentage of the respective $25 \mathrm{mM}$ glucose-treated cells. Data are expressed as mean \pm SEM and analyzed using oneway ANOVA with LSD analysis. ${ }^{\Delta} P<0.05,{ }^{\Delta \Delta} P<0.01$, compared to $25 \mathrm{mM}$ glucose; ${ }^{\# \#} P<0.01$, compared to $150 \mathrm{mM}$ glucose; ${ }^{\star} P<0.05,{ }^{\star}{ }^{\star} P<0.01$, Student's unpaired t-test compared to 24 hours' time point. ${ }^{*} P<0.05$, $* * P<0.01(\mathrm{n}=4$ per group). 

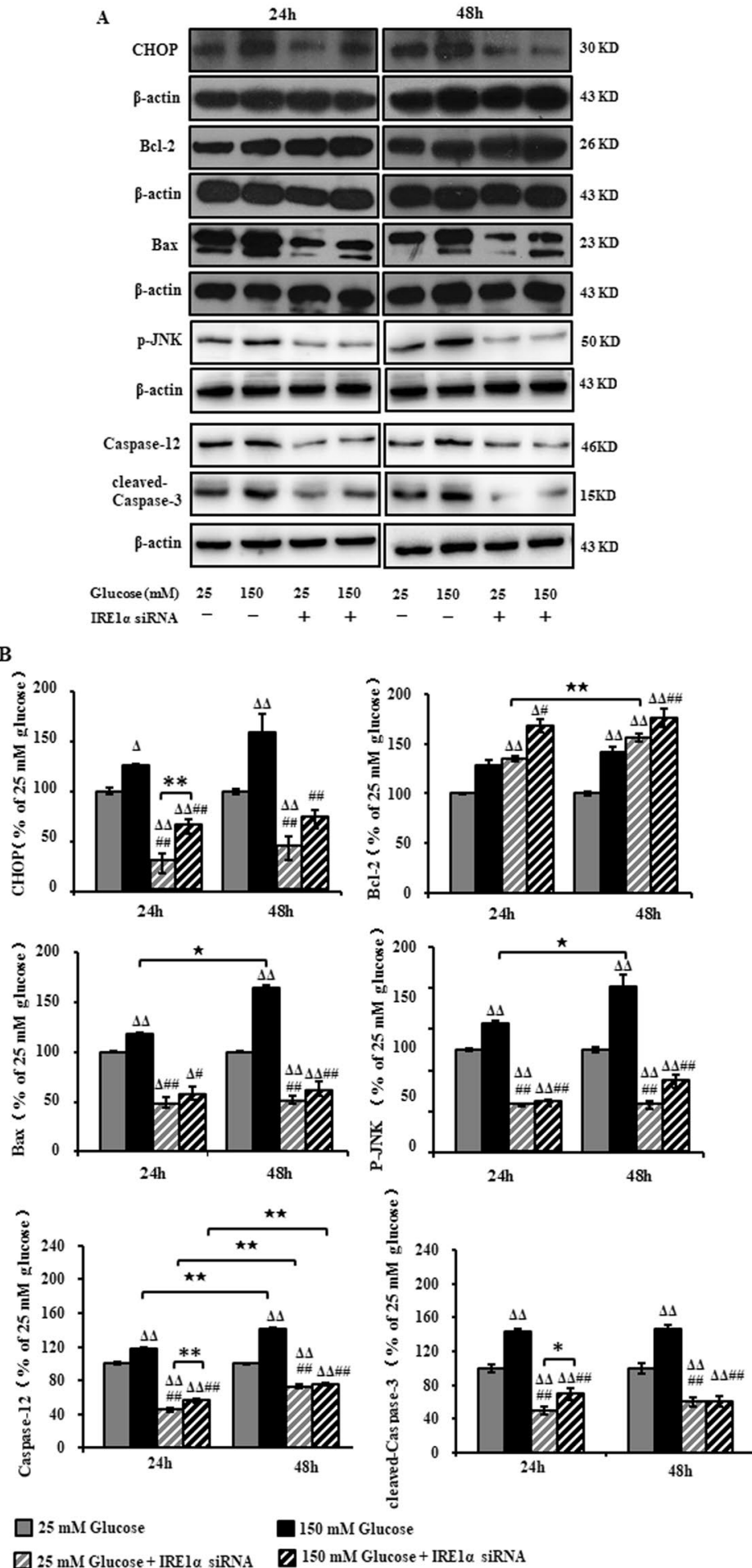

Z $25 \mathrm{mM}$ Glucose + IREl $\alpha$ siRNA

$150 \mathrm{mM}$ Glucose

Z $150 \mathrm{mM}$ Glucose + IREl $\alpha$ siRNA

Figure 6. Expression of CHOP, Bcl-2, Bax, p-JNK, Caspase-12 and cleaved-Caspase- 3 in RSC96 cells after IRE1 $\alpha$ siRNA transfection and exposure to high glucose. (A) Representative Western blots using tissue extracts from the sciatic nerve and probed with antibodies against CHOP, Bcl-2, Bax, p-JNK, Caspase-12 and cleavedCaspase-3. (B) The band intensity expressed as mean \pm SEM of the percentage of the respective $25 \mathrm{mM}$ glucosetreated cells and analyzed using one-way ANOVA with LSD analysis (CHOP, Bcl-2/48 h, Bax/48 h, p-JNK/24 h, Caspase-12, cleaved-Caspase-3,) or Tamhane's T2 analysis (p-JNK/48 h, Bcl-2/24 h, Bax/24h, Caspase-3/24h,). ${ }^{\Delta} P<0.05,{ }^{\Delta \Delta} P<0.01$, compared to $25 \mathrm{mM}$ glucose; ${ }^{\#} P<0.05$, ${ }^{\#} P<0.01$, compared to $150 \mathrm{mM}$ glucose; ${ }^{\star} P<0.05,{ }^{\star}{ }^{\star} P<0.01$, Student's unpaired t-test compared to 24 hours' time point. $* P<0.05, * * P<0.01$ ( $\mathrm{n}=4$ per group). 


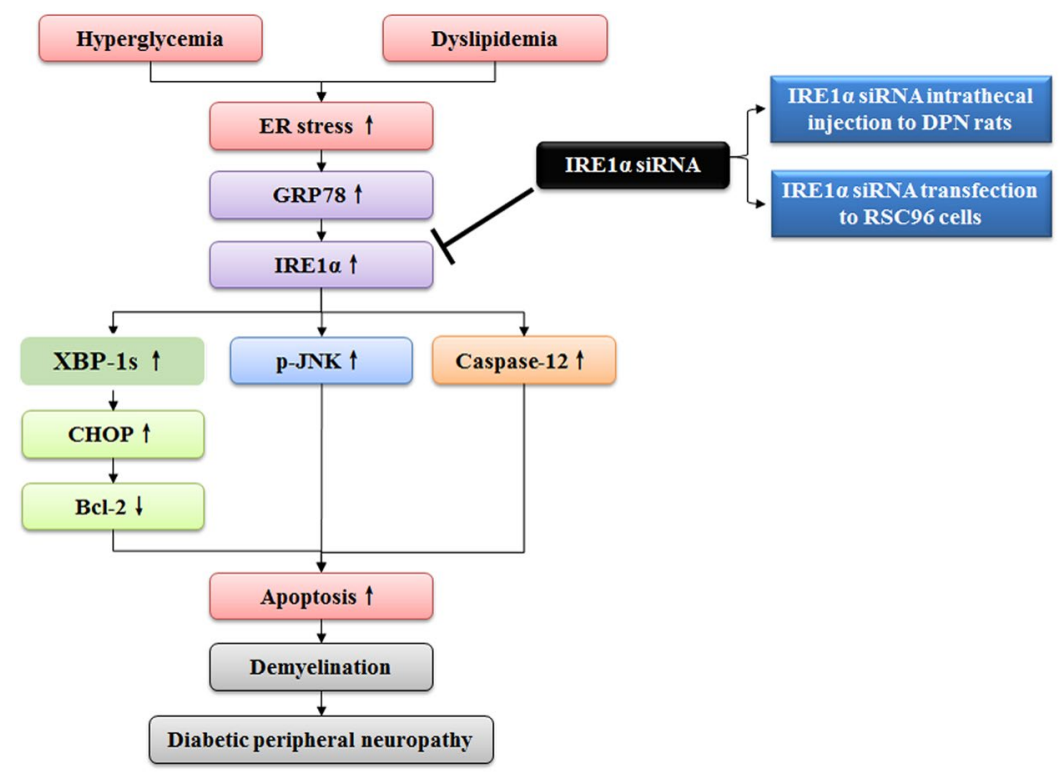

Figure 7. Schematic diagram depicting improvement of diabetic peripheral neuropathy due to relieved endoplasmic reticulum stress-induced apoptosis by IRE1 $\alpha$ siRNA.

In the sciatic nerve of DPN rats and SCs exposed to high glucose, GRP78 was up-regulated ${ }^{7}$. GRP78 is triggered and overexpressed when ER stress occurs ${ }^{14}$. Our results showed that inhibition of IRE1 $\alpha$ didn't change the expression of GRP78, suggesting that the inhibition may alter the status of ER stress in DPN and alleviate DPN through reducing the expression of IRE1 $\alpha$-induced apoptosis genes.

Recently, targeting the IRE1 $\alpha$ pathway has emerged as a potential approach for treatment of diabetes and its complications ${ }^{18}$. The ER is the main organelle for calcium storage, protein folding and processing, as well as for lipid biosynthesis and metabolism. Metabolic perturbations such as advanced glycation of proteins and lipids, oxidative stress, that are often seen in diabetes and related disorders, lead to ER stress. ER stress activates UPR signaling pathways that determine cell fate.

Remediable ER stress activates adaptive-UPR outputs that favor cell survival. A major advance in understanding the involvement of ER stress in the pathobiology of metabolic disorders has been the discovery of the XBP1, a modulator of glucose and lipid metabolism ${ }^{12}$. Spliced X-box binding protein 1 (sXBP1) translocates to the nucleus to initiate transcription of chaperone proteins and proteins involved in ER-associated protein degradation (ERAD). These adaptive mechanisms of the UPR function to attenuate mild to moderate ER stress to restore normal ER function.

But chronic ER stress activates T-UPR outputs will trigger apoptosis. During times of extreme or chronic stress, for example sustained hyperglycemia, the capacity of the UPR is overwhelmed, induces the T-UPR, and drive IRE1a autophosphorylation and XBP1 mRNA splicing. The IRE1 $\alpha /$ XBP-1 pathway is dominant to promote apoptosis, ${ }^{6,11,13}$.

Our results show that CHOP was upregulated in the DPN rats and Schwann cells and IRE1 $\alpha$ inhibition suppressed the CHOP expression. CHOP plays a critical role in ER stress-induced apoptosis and it is believed to play a central role in ER stress-induced cell death. It is strongly induced via IRE1 signaling ${ }^{19}$.

In addition, IRE1 $\alpha$ triggers several other proapoptotic signaling pathways, including ER-resident Caspase- 12. Caspase-12 is located on the ER and activated in ER stress-induced apoptosis, but not by membrane- or mitochondrial-targeted apoptotic signals. It has been shown to be important in apoptosis because it is elicited by ER stress in neurodegenerative disease ${ }^{20,21}$. Our present study shows that the inhibition of IRE1 $\alpha$ markedly decreased Caspase-12. Previous studies have demonstrated that cleaved-Caspase- 3 is involved in ER stress-induced apoptosis and is activated by Caspase-12. Our observations confirmed that cleaved-Caspase- 3 was markedly reduced when Caspase- 12 was downregulated as a result of IRE1 $\alpha$ siRNA transfection.

IRE $1 \alpha$ forms a complex with TRAF2 and ASK1, and in turn it recruits and activates JNK ${ }^{14}$. The activation of JNK pathway has been shown to be a common phenomenon in stress-induced apoptosis in response to intracellular stresses and activates $\mathrm{Bax}^{22}$. In this research, IRE1 $\alpha$ inhibition resulted in reduced JNK activation and then suppressed Bax expression. In addition, $\mathrm{CHOP}$ also down regulates $\mathrm{Bcl}-2$ expression and increases the translocation of Bax from the cytosol to mitochondria, which consequently triggers the activation of caspase-3, resulting in apoptosis ${ }^{23}$. Taken together, ER stress-induced apoptosis is relieved by IRE1 $\alpha$ inhibition ${ }^{24}$.

In this study, we investigated IRE1 $\alpha$ related-apoptosis induced by ER stress in DPN both in vivo and in vitro to elucidate the effect of IRE1 $\alpha$ siRNA on DPN. Our data highlight that IRE1 $\alpha$ siRNA reduces the CHOP, Caspase-12 and JNK activation-related apoptosis, decreases the demyelination, thereby improving the DPN (Fig. 7), suggesting that IRE1 $\alpha$ is a potentially valuable target for interventions aimed at improving DPN.

\section{Methods}

Animals and cells. Animal experiments procedures were approved by the Animal Care and Use Committee of the Capital Medical University (approval no. AEEI-2014-086). Male Sprague-Dawley (SD) rats weighing 
180-220 g were obtained from the Experimental Animal Center at Capital Medical University, Beijing, China (SCXK 2012-0001) and were housed with a 12/12-hour light/dark cycle in a temperature and humidity-controlled environment. All experiments were performed in accordance with relevant guidelines and regulations.

RSC96 cells (CRL-2765) were purchased from the American Type Culture Collection (ATCC, cat no. CRL2765 ) and were cultured in DMEM modified to contain $4 \mathrm{mM} \mathrm{L}$-glutamine, $25 \mathrm{mM}$ glucose, $1 \mathrm{mM}$ sodium pyruvate, $1500 \mathrm{mg} / \mathrm{L}$ sodium bicarbonate, $10 \% \mathrm{FBS}$ and $1 \%$ antibiotics at $37^{\circ} \mathrm{C}$ in a humidified atmosphere of $5 \% \mathrm{CO}_{2}$ and were passaged once every 3 days.

Animal model of DPN and IRE1 $\alpha$ siRNA intrathecal injection. Animals were divided into control, DPN, control plus IRE1 $\alpha$ siRNA and DPN plus IRE1 $\alpha$ siRNA groups. DPN was induced by feeding the animals with high-carbohydrate/high-fat diet and low-dose streptozotocin (STZ, S0130, Sigma). Specifically, rats in two DPN groups were fed with high-carbohydrate/high-fat diet (67\% standard diet, $10 \%$ lard, $20 \%$ sucrose, $2.5 \%$ cholesterol and $0.5 \%$ cholate) for 4 weeks followed by treating with streptozotocin $(35 \mathrm{mg} / \mathrm{kg}$ in $0.1 \mathrm{M}$ citric acid buffer, $\mathrm{pH} 4.5$, i.p.) The rats with fasting blood glucose level above $16.7 \mathrm{mM}$ 1-week after STZ injection were considered diabetic and were continued feeding on the high-carbohydrate/high-fat diet for 12 weeks. Fasting blood glucose levels were measured every 2 weeks to monitor the persistence of diabetes and thermal perception thresholds (TPT) were measured every 4 weeks to track the occurrence of peripheral neuropathy.

At 12 weeks after induction of diabetes, intrathecal injection of IRE1 siRNA was carried out. Rats were anesthetized using chloral hydrate (i.p., $300 \mathrm{mg} / \mathrm{kg}$ ). L4-L5 vertebrae were exposed and a sterile polyethylene tubing (PE-10 catheter) was implanted into the subarachnoid space according to the previously published ${ }^{25}$. Rats were allowed 24 hours to recover from surgery prior to treatment.

IRE1 $\alpha$ siRNA (sc-270028, Santa Cruz, USA) was prepared immediately prior to administration by mixing the siRNA solution with a transfection regent (sc-29528, Santa Cruz, USA), in a ratio of $1: 4(\mathrm{v} / \mathrm{v})$. The final concentration of RNA as an RNA/lipid complex was $0.4 \mu \mathrm{g} / \mu \mathrm{L}$. The IRE $1 \alpha$ siRNA complex or transfection regent $(10 \mu \mathrm{L})$ alone was injected in Control IRE1 $\alpha$ siRNA transfected (Control + IRE1 $\alpha$ siRNA), DPN IRE1 $\alpha$ siRNA transfected (DPN + IRE1 $\alpha$ siRNA) and Control, DPN groups using a $25 \mu \mathrm{L}$ microsyringe, respectively. $10 \mu \mathrm{L}$ saline solution was used to wash the catheter. Injections were given daily for 3 consecutive days ${ }^{24,26}$. Nerve conduction velocity test and blood, tissue harvest were carried out 24 hour after the last injection.

Cells culture and transfection. RSC96 cells plated at a density of $2 \times 10^{5}$ cells/well in 6 -well plate were allowed to adhere overnight, and then transfected with IRE1 $\alpha$ siRNA according to the manufacturer's instructions. Briefly, for each transfection, 100 pmols of siRNA and $6 \mu$ transfection reagent were added to $100 \mu \mathrm{l}$ siRNA transfection medium (sc-36868, Santa Cruz), gently mixed, incubated for 25 minutes at room temperature, then $1.0 \mathrm{ml}$ siRNA transfection medium containing $200 \mu \mathrm{l}$ siRNA transfection reagent mixture was added to the well. Cells were incubated 8 hours at $37^{\circ} \mathrm{C}$ in a $\mathrm{CO}_{2}$ incubator, $1 \mathrm{ml}$ normal growth medium containing 2 times the normal serum and antibiotics concentration $(2 \times$ normal growth medium) was added without removing the transfection mixture. Cells were incubated for an additional 16 hours and the medium was aspirated and cultured in $25 \mathrm{mM}$ or $150 \mathrm{mM}$ glucose growth medium for 24 hours and 48 hours, respectively.

Cells were treated with $25 \mathrm{mM}$ glucose, $150 \mathrm{mM}$ glucose, $25 \mathrm{mM}$ glucose IRE1 $\alpha$ siRNA transfected $(25 \mathrm{mM}$ glucose + IRE1 $\alpha$ siRNA) and $150 \mathrm{mM}$ glucose IRE1 $\alpha$ siRNA transfected ( $150 \mathrm{mM}$ glucose + IRE1 $\alpha$ siRNA). Cells in $25 \mathrm{mM}$ glucose, $150 \mathrm{mM}$ glucose groups were treated with siRNA transfection reagent alone (Supplementary Fig. 1).

Measurement of nerve conduction velocity. Nerve conduction velocity was assessed using Functional Experiment System (BL-420s, Techman, China) as reported previously ${ }^{27}$. Before the measurement, rats were anesthetized with $10 \%$ chloral hydrate (i.p., $300 \mathrm{mg} / \mathrm{kg}$ ). For measurement of MNCV, stimulation electrode was placed at the notch of sciatic nerve. Sciatic nerve was stimulated with single square wave pluses $(1.2 \mathrm{~V}$ in intensity, $1 \mathrm{~ms}$ in width). For SNCV, recording site was located in the sciatic notch. Stimulation parameters were the same as MNCV methods.

Morphometric analysis of sciatic nerve. After the conduction velocity test, the left sciatic nerve was isolated and cut into two segments. One segment $(2 \mathrm{~mm})$ was fixed in $2.5 \%$ glutaraldehyde at $4{ }^{\circ} \mathrm{C}$, and sent to the Electron Microscopy Center of Institute of Capital Medical University for ultrastructure observation.

The other segment $(1 \mathrm{~cm})$ was fixed in $10 \%$ buffered formalin and processed for paraffin section. Sciatic nerve tissues were cut in slice of $5 \mu \mathrm{m}$ thick. Sections were stained with Luxol fast blue, and microphotographs were captured using a light microscope (Nikon Eclipse 80i, Japan).

Terminal dUTP nick-end labeling (TUNEL) assay. Apoptotic tissues were labeled using in situ cell death detection kit from Roche Company, according to manufacturer's protocol. The nerve sections were dewaxed and followed by incubation with proteinase $\mathrm{K}$ for $15 \mathrm{~min}$ at room temperature. Following digestion, the end-labeling reaction was performed by adding TdT and dUT reaction mix onto the slides at $37^{\circ} \mathrm{C}$ for $1 \mathrm{~h}$ at humidified chamber. After three PBS washes, converter-POD was added onto sections and incubated at $37^{\circ} \mathrm{C}$ for $30 \mathrm{~min}$ at humidified chamber followed by reaction with $\mathrm{DAB}$ at room temperature for $10 \mathrm{~min}$. Staining was observed using a light microscope.

Immunofluorescence staining. The nerve sections were dewaxed, antigen repaired and blocked with 3\% BSA. One hour later, sections were incubated in the following primary antibodies overnight at $4{ }^{\circ} \mathrm{C}$ : anti-IRE $1 \alpha$ (sc-390960; 1: 50; Santa Cruz), anti-p-JNK (sc-6254; 1: 50; Santa Cruz) and rabbit anti-Caspase-12 (sc-5627; 1: 50; Santa Cruz). After rinsing, the sections were incubated with the fluorescein (FITC)-conjugated goat anti-mouse $\operatorname{IgG}(\mathrm{H}+\mathrm{L})$ or FITC-conjugated goat anti-rabbit $\operatorname{IgG}(\mathrm{H}+\mathrm{L})$ for $1 \mathrm{~h}$ at room temperature. Microphotographs were captured using the Nikon Eclipse 80i light microscope ${ }^{7}$. 
Western blotting. Sciatic nerves and RSC96 cells were lysed on ice in the RIPA buffer with protease inhibitor cocktail and phosphatase inhibitor cocktail for $30 \mathrm{~min}$ to extract total proteins. The proteins were analyzed with a bicinchoninic acid (BCA) protein assay kit (Biosynthesis, China). $30 \mu \mathrm{g} / \mathrm{lane}$ (sciatic nerve) and $20 \mu \mathrm{g} /$ lane (RSC96 cell) were used for Western blot analysis as previously described ${ }^{8,27}$. The primary antibodies were as follows: mouse anti-IRE1 $\alpha$ (sc-390960; 1: 1000; Santa Cruz), rabbit anti-P-IRE1 $\alpha$ (ab48187; 1: 2000; abcam), mouse anti-XBP1 (sc-8015; 1: 1000; Abcam), mouse anti-GRP78 (sc-376768; 1: 1000; Santa Cruz), mouse anti-GADD153 (sc-7351; 1: 500; Santa Cruz), rabbit anti-Caspase-3 (ab13847; 1: 1000; abcam), rabbit anti-Caspase-12 (om273459; 1: 1000; Omnimabs), mouse anti-Bcl-2 (sc-7382; 1: 1000; Santa Cruz), mouse anti-Bax (sc-7480; 1: 1000; Santa Cruz), mouse anti-p-JNK (sc-6254; 1: 1000; Santa Cruz), and rabbit anti-PGP9.5 (ab108986; 1: 2000; Abcam). Mouse anti- $\beta$-actin (TA-09; 1: 20000; Zhongshan Goldenbridge) served as the internal control. The secondary antibodies were goat anti-mouse IgG-HRP (ZB-2305; Zhongshan Goldenbridge) and goat anti-rabbit IgG-HRP (ZB-2301; Zhongshan Goldenbridge). Western Chemiluminescent HRP Substrate and exposed to X-film to form image. The protein bands were quantitated with Image J software ${ }^{27}$.

Apoptosis assay. An Annexin-FITC Apoptosis Detection Kit (AP101, Multisciences, China) was used to examine apoptosis according to the manufacturer's instructions ${ }^{8}$. In brief, cells were added with $500 \mu \mathrm{L}$ of binding buffer followed by staining with $5 \mu \mathrm{L}$ of FITC-labeled annexin $\mathrm{V}$ and $10 \mu \mathrm{L}$ of propidium iodide (PI) and incubated at room temperature for $5 \mathrm{~min}$ in the dark. BD LSRFortessa ${ }^{\mathrm{TM}}$ flow cytometer (BD Biosciences, San Jose, CA, USA) were used to analysis.

Intracellular $\mathrm{Ca}^{2+}$ analysis. Fluo-3 AM (S1056, Beyotime, China) was used to examine intracellular $\mathrm{Ca}^{2+}$ levels according to the manufacturer's instructions ${ }^{28}$. Cells were harvested and washed twice with PBS, then loaded with Fluo-3 AM $(5 \mu \mathrm{M})$ for $30 \mathrm{~min}$ at $37^{\circ} \mathrm{C}$ in the dark, and was analyzed using BD LSRFortessa ${ }^{\mathrm{TM}}$ flow cytometer.

Statistical analysis. Data were expressed as the mean \pm SEM. Statistical analysis was performed by unpaired Student's t-test or One-way ANOVA followed by least significant difference (LSD) or Tamhane's T2 analysis using SPSS 17.0. Values of $P<0.05$ were statistically significant.

Data availability. The datasets generated during and/or analyzed during the current study are available from the corresponding author on reasonable request.

\section{References}

1. Callaghan, B. C., Cheng, H. T., Stables, C. L., Smith, A. L. \& Feldman, E. L. Diabetic neuropathy: clinical manifestations and current treatments. The Lancet Neurology 11, 521-534, https://doi.org/10.1016/s1474-4422(12)70065-0 (2012).

2. Goncalves, N. P. et al. Schwann cell interactions with axons and microvessels in diabetic neuropathy. Nature reviews. Neurology 13, 135-147, https://doi.org/10.1038/nrneurol.2016.201 (2017).

3. Valenzuela, V., Onate, M., Hetz, C. \& Court, F. A. Injury to the nervous system: A look into the ER. Brain research 1648, 617-625, https://doi.org/10.1016/j.brainres.2016.04.053 (2016).

4. Pareyson, D., Piscosquito, G., Moroni, I., Salsano, E. \& Zeviani, M. Peripheral neuropathy in mitochondrial disorders. Lancet Neurol 12, 1011-1024, https://doi.org/10.1016/S1474-4422(13)70158-3 (2013)

5. Chen, Y. \& Brandizzi, F. IRE1: ER stress sensor and cell fate executor. Trends in cell biology 23, 547-555, https://doi.org/10.1016/j. tcb.2013.06.005 (2013)

6. Morita, S. et al. Targeting ABL-IRE1alpha Signaling Spares ER-Stressed Pancreatic beta Cells to Reverse Autoimmune Diabetes. Cell metabolism 25, 883-897 e888, https://doi.org/10.1016/j.cmet.2017.03.018 (2017).

7. Yang, X. et al. Tangluoning, a traditional Chinese medicine, attenuates in vivo and in vitro diabetic peripheral neuropathy through modulation of PERK/Nrf2 pathway. Scientific reports 7, 1014, https://doi.org/10.1038/s41598-017-00936-9 (2017).

8. Yang, X. et al. Paeoniflorin protects Schwann cells against high glucose induced oxidative injury by activating Nrf2/ARE pathway and inhibiting apoptosis. Journal of ethnopharmacology 185, 361-369, https://doi.org/10.1016/j.jep.2016.03.031 (2016).

9. Kim, D. H. IRE1 Sulfenylation by Reactive Oxygen Species Coordinates Cellular Stress Signaling. Molecular cell 63, 541-542, https:// doi.org/10.1016/j.molcel.2016.08.003 (2016).

10. Argemi, J. et al. X-box Binding Protein 1 Regulates Unfolded Protein, Acute-Phase, and DNA Damage Responses During Regeneration of Mouse Liver. Gastroenterology 152, 1203-1216 e1215, https://doi.org/10.1053/j.gastro.2016.12.040 (2017).

11. Eletto, D., Eletto, D., Boyle, S. \& Argon, Y. PDIA6 regulates insulin secretion by selectively inhibiting the RIDD activity of IRE1. FASEB J 30, 653-665, https://doi.org/10.1096/fj.15-275883 (2016).

12. So, J. S. et al. Silencing of lipid metabolism genes through IRE1alpha-mediated mRNA decay lowers plasma lipids in mice. Cell metabolism 16, 487-499, https://doi.org/10.1016/j.cmet.2012.09.004 (2012).

13. Gao, H. H. et al. Autophagy inhibition of immature oocytes during vitrification-warming and in vitro mature activates apoptosis via caspase-9 and -12 pathway. European Journal of Obstetrics \& Gynecology and Reproductive Biology 217, 89-93, https://doi. org/10.1016/j.ejogrb.2017.08.029 (2017).

14. O’Brien, P. D., Hinder, L. M., Sakowski, S. A. \& Feldman, E. L. ER stress in diabetic peripheral neuropathy: A new therapeutic target. Antioxidants \& redox signaling 21, 621-633, https://doi.org/10.1089/ars.2013.5807 (2014).

15. Shi, H. T. et al. Effect of Tangluoning on PERK-CHOP-Caspase-12 pathway in diabetic peripheral neuropathy rats. Global Traditional Chinese Medicine 10, 269-274 (2017).

16. Feldman, E. L., Nave, K. A., Jensen, T. S. \& Bennett, D. L. New Horizons in Diabetic Neuropathy: Mechanisms, Bioenergetics, and Pain. Neuron 93, 1296-1313, https://doi.org/10.1016/j.neuron.2017.02.005 (2017).

17. Clayton, B. L. \& Popko, B. Endoplasmic reticulum stress and the unfolded protein response in disorders of myelinating glia. Brain research 1648, 594-602, https://doi.org/10.1016/j.brainres.2016.03.046 (2016).

18. Piperi, C., Adamopoulos, C. \& Papavassiliou, A. G. XBP1: A Pivotal Transcriptional Regulator of Glucose and Lipid Metabolism. Trends in endocrinology and metabolism: TEM 27, 119-122, https://doi.org/10.1016/j.tem.2016.01.001 (2016).

19. Mihailidou, C., Papavassiliou, A. G. \& Kiaris, H. A crosstalk between p21 and UPR-induced transcription factor C/EBP homologous protein (CHOP) linked to type 2 diabetes. Biochimie 99, 19-27, https://doi.org/10.1016/j.biochi.2013.11.003 (2014).

20. Li, L. et al. Hyperglycemia magnifies bupivacaine-induced cell apoptosis triggered by mitochondria dysfunction and endoplasmic reticulum stress. Journal of neuroscience research 91, 786-798, https://doi.org/10.1002/jnr.23216 (2013). 
21. Tong, Q. et al. Inhibition of endoplasmic reticulum stress-activated IRE1alpha-TRAF2-caspase-12 apoptotic pathway is involved in the neuroprotective effects of telmisartan in the rotenone rat model of Parkinson's disease. European journal of pharmacology 776, 106-115, https://doi.org/10.1016/j.ejphar.2016.02.042 (2016).

22. Zeng, T. et al. IRE1alpha-TRAF2-ASK1 complex-mediated endoplasmic reticulum stress and mitochondrial dysfunction contribute to CXC195-induced apoptosis in human bladder carcinoma T24 cells. Biochemical and biophysical research communications 460 , 530-536, https://doi.org/10.1016/j.bbrc.2015.03.064 (2015).

23. McCullough, K. D., Martindale, J. L., Klotz, L. O., Aw, T. Y. \& Holbrook, N. J. Gadd153 sensitizes cells to endoplasmic reticulum stress by down-regulating Bcl2 and perturbing the cellular redox state. Mol Cell Biol 21, 1249-1259, https://doi.org/10.1128/ MCB.21.4.1249-1259.2001 (2001)

24. Luo, M. C. et al. An efficient intrathecal delivery of small interfering RNA to the spinal cord and peripheral neurons. Molecular pain 1, 29, https://doi.org/10.1186/1744-8069-1-29 (2005).

25. Chen, J.-J. et al. Intrathecal curcumin attenuates pain hypersensitivity and decreases spinal neuroinflammation in rat model of monoarthritis. Sci. Rep. 5, 10278, https://doi.org/10.1038/srep10278 (2015)

26. Wu, Y.-B. et al. CHOP/ORP150 Ratio in Endoplasmic Reticulum Stress: A New Mechanism for Diabetic Peripheral Neuropathy. Cellular Physiology and Biochemistry 32, 367-379, https://doi.org/10.1159/000354444 (2013).

27. Yang, X. et al. Mechanism of Tang Luo Ning effect on attenuating of oxidative stress in sciatic nerve of STZ-induced diabetic rats. Journal of ethnopharmacology 174, 1-10, https://doi.org/10.1016/j.jep.2015.07.047 (2015).

28. Yao, W. et al. Tang-Luo-Ning, a Traditional Chinese Medicine, Inhibits Endoplasmic Reticulum Stress-Induced Apoptosis of Schwann Cells under High Glucose Environment. Evidence-Based Complementary and Alternative Medicine, 2017, Article ID5193548, https://doi.org/10.1155/2017/5193548 (2017).

\section{Acknowledgements}

This work was supported by National Natural Science Foundation of China (grant No. 81473642) and Capital Research of Traditional Chinese Medicine (Grant No. 17ZY19).

\section{Author Contributions}

X.Y. and L.X. designed the research, and contributions equally to this paper; W.Y., X.Y., J.Z., B.G. and H.S. performed experiments, W.Y., B.G. and H.S. supervised the in vitro Schwann cell model work, W.Y. and J.Z. supervised the in vivo DPN cell model work; X.Y. and W.Y. analyzed data; X.Y., W.Y. and L.X. interpreted the results; J.Z., B.G. and W.Y. wrote the manuscript. All authors read and approved the final manuscript.

\section{Additional Information}

Supplementary information accompanies this paper at https://doi.org/10.1038/s41598-018-20950-9.

Competing Interests: The authors declare no competing interests.

Publisher's note: Springer Nature remains neutral with regard to jurisdictional claims in published maps and institutional affiliations.

(c) (i) Open Access This article is licensed under a Creative Commons Attribution 4.0 International

License, which permits use, sharing, adaptation, distribution and reproduction in any medium or format, as long as you give appropriate credit to the original author(s) and the source, provide a link to the Creative Commons license, and indicate if changes were made. The images or other third party material in this article are included in the article's Creative Commons license, unless indicated otherwise in a credit line to the material. If material is not included in the article's Creative Commons license and your intended use is not permitted by statutory regulation or exceeds the permitted use, you will need to obtain permission directly from the copyright holder. To view a copy of this license, visit http://creativecommons.org/licenses/by/4.0/.

(c) The Author(s) 2018 